\title{
Real-time 3D Ultrasound-based Servoing of a Surgical Instrument
}

\author{
Jeffrey Stoll ${ }^{1}$, Paul Novotny ${ }^{2}$, Robert Howe ${ }^{2}$, and Pierre Dupont ${ }^{1}$ \\ ${ }^{1}$ Aerospace and Mechanical Engineering Department \\ Boston University, Boston, MA \\ \{jstoll,pierre\}@bu.edu \\ ${ }^{2}$ Division of Engineering and Applied Science \\ Harvard University, Cambridge, MA \\ novotny@fas.harvard.edu,howe@deas.harvard.edu
}

\begin{abstract}
This paper presents a real-time 3D ultrasoundguided robotic system, designed to autonomously navigate a surgical instrument to surgeon-specified target points. The system tracks the instrument in real-time $(2 \mathrm{~Hz})$ using image processing. Thus, it does not require tracking of the ultrasound scan head. It makes use of a line detection algorithm and a passive instrument marker, which together report the instrument's position and orientation (6 d.o.f.) from a single ultrasound image. Validation experiments are presented. The system controlled instrument position in a variety of image locations with a mean error of $0.8 \mathrm{~mm}$.
\end{abstract}

Index Terms - robotic surgery, ultrasound, visual servoing

\section{INTRODUCTION}

Recent studies have shown the potential of ultrasound imaging for guiding minimally invasive surgical interventions when direct vision is not possible. For example, in beatingheart intra-cardiac surgery a surgeon must work inside the heart while it is filled with opaque blood, thus precluding the use of an endoscopic camera [1]. With the advent of real-time 3D ultrasound, it is now possible to perform this kind of procedure.

A drawback of ultrasound imaging, however, is its low signal to noise ratio. As a result, small tissue structures can be difficult to identify and track manually, especially if they are moving. In addition, surgical instruments and tissue are substantially different in both their impedance and absorption of sound. This difference produces image artifacts, which obscure the instrument's location and geometric detail. For example, Figure 1 shows a 3D ultrasound image of a typical surgical grasper, where image artifacts have significantly obscured the grasping fingers. These challenges hinder a surgeon's ability to navigate instruments accurately.

Several researchers have studied ultrasound guidance of surgical robotic systems. A robotic system to perform diagnostic $2 \mathrm{D}$ ultrasound scans, using visual servoing to track tissue structures is reported in [2]. In this case, the robot manipulated the ultrasound scan head, rather than an instrument. A robotic system for needle insertion under 2D ultrasound guidance, which compensates for patient movement, is reported in [3]. In this case, the position and

This research was funded by the NIH under Bioengineering Research Partnership grant \#R01-HL073647.

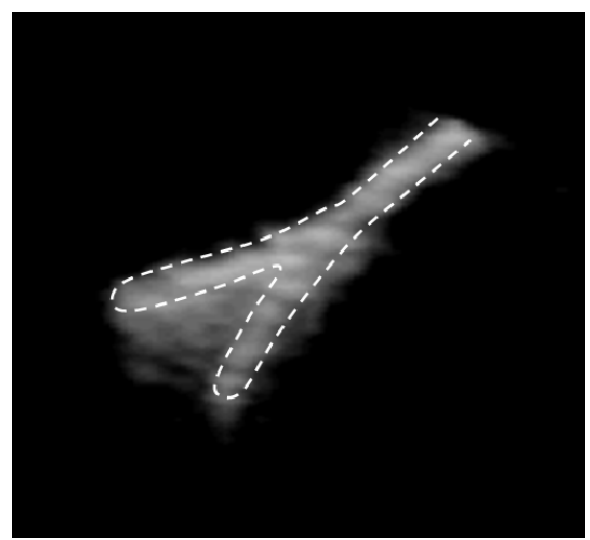

Fig. 1 - 3D ultrasound close-up image of a surgical grasper. Grasping fingers are outlined.

orientation of the needle and target in the image are determined automatically. The needle is mechanically constrained to lie in the ultrasound scan plane but is actuated in its remaining degrees of freedom. Measurements of grasper finger location in 2D ultrasound images are used to guide a robot to specified target points in [4]. This system requires the scan plane to be oriented roughly perpendicular to the tracked instrument. Active tracking devices have also been investigated. For example, tracking of an active ultrasound receiver mounted on a cardiac catheter using 2D imaging is reported in [5][6]. The same approach using realtime 3D imaging is presented in [7]. Although this approach produces high accuracy, it requires the instrument to be integrated electronically with the ultrasound scanner, which adds cost and complexity. Finally, the use of commercial electromagnetic and optical tracking systems have been investigated [8][9][10]. These systems make use of tracking receivers mounted on both the ultrasound scan head and instruments/robot. These receivers report the ultrasound image and instrument positions and orientations with respect to a fixed base frame. This approach requires complex calibration, however, which introduces errors into the tracking result.

This paper presents a 3D ultrasound image-guided robotic system designed to autonomously navigate a minimally invasive surgical instrument in 6 d.o.f, using only passive tracking. To the authors' knowledge, this is the first example of such a system using real-time 3D ultrasound. Real-time instrument tracking is performed by a combination of two 


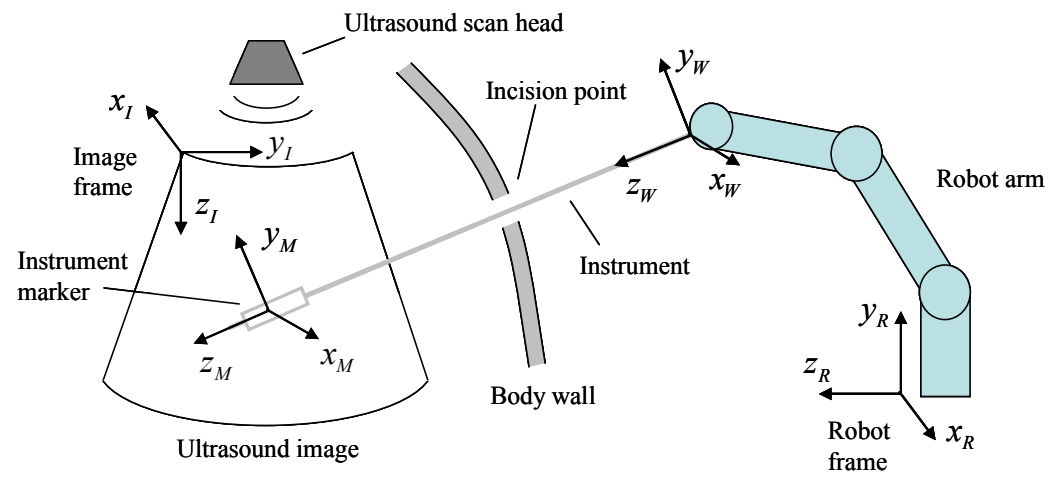

a)

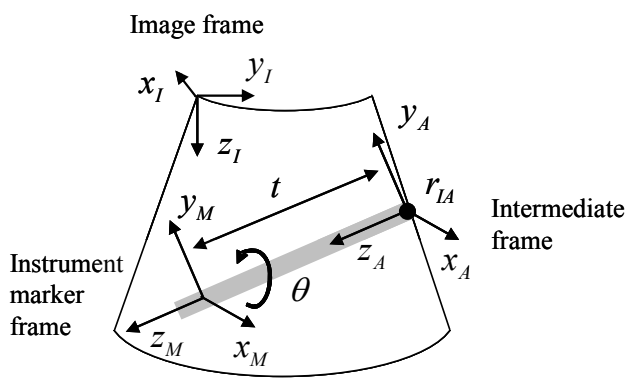

b)

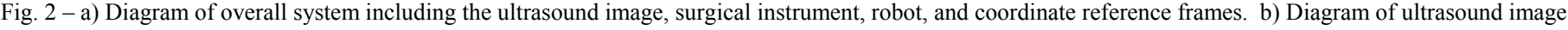
showing intermediate frame.

techniques. First, the instrument shaft axis is measured by 3D line detection, based on the singular value decomposition [11][12]. Second, a passive marker is used to determine the position and orientation of the instrument tip [13]. The marker and instrument shaft together indicate the position and orientation of the instrument using a single 3D ultrasound volume.

\section{SYSTEM DESCRIPTION}

Figure 2a shows a diagram of the complete system. It consists of a real-time 3D ultrasound scanner imaging the instrument with the marker attached. The instrument is controlled outside the body by a robot arm and passes into the body through a small incision.

The instrument is controlled by measuring the tip position error in the image. This error measurement is then transformed to robot coordinates via $T_{R}^{I}$, the transformation between the ultrasound image frame and the robot base frame,

$$
T_{R}^{I}=T_{R}^{W} T_{W}^{M} T_{M}^{I}
$$

This process is commonly referred to as registration. The term $T_{M}^{I}$ is the transformation from the image frame to a local frame on the instrument, defined by the instrument shaft and marker as described in sections IIa and b. The term $T_{W}^{M}$ is the transformation between this instrument frame and a 3 d.o.f. wrist mounted on the robot. It is determined in an initial calibration step and remains fixed since the marker is rigidly attached to the instrument. Finally, the term $T_{R}^{W}$ is the transformation between the gimbal endpoint and the robot base frame. It is measured via the robot joint encoders. Image pixel scaling is determined offline so that all coordinate frames have units of $\mathrm{mm}$. The location of a surgical target is then given in robot coordinates by

$$
p_{R}=T_{R}^{I} p_{I},
$$

where $p_{I}$ is the target location in image coordinates.

The incision in the body wall (port) acts like a fulcrum as it supports the instrument. This forms a constraint on the instrument's motion. Therefore, a goal point for the robot wrist is determined by projecting the target point through the port according to,

$$
p_{W}=p_{R}+\delta \frac{p_{R}-b_{R}}{\left\|p_{R}-b_{R}\right\|},
$$

where $b_{R}$ is the location of the port in robot coordinates and $\delta$ is the length of the instrument. The robot wrist is then commanded to the point $p_{W}$ so that the instrument tip approaches the target.

The following subsections illustrate the details of instrument tracking. In particular, the transformation $T_{M}^{I}$ can be decomposed into two elements, one identified via the image of the instrument shaft and the other via the instrument marker,

$$
T_{M}^{I}=T_{M}^{A} T_{A}^{I}
$$

As shown in Figure $2 \mathrm{~b}$, transformation $T_{A}^{I}$ relates an intermediate frame, $A$, located on the instrument shaft, to the image frame. Frame $A$ is defined such that $z_{A}$ corresponds to the shaft axis and points toward the instrument tip and $y_{A}$ lies in the vertical plane parallel to $z_{A}$. The second transformation, $T_{M}^{A}(\theta, t)$, relates the local instrument frame with respect to $A$ in terms of $\theta$ and $t$, the rotation about, and the translation along, the shaft axis $z_{A}$.

\section{A. Instrument Shaft Tracking}

In an ultrasound image, the instrument shaft appears as a line of high pixel intensity. This line represents the surface of the instrument shaft facing the ultrasound transducer. Its configuration can be described with 4 parameters $(\eta, \mu, \phi, \psi)$. The terms $\eta$ and $\mu$ are the coordinates of the instrument shaft in the planar subspace orthogonal to it, and the terms $\phi$ and $\psi$ are Euler angles describing the instrument axis, $z_{A}$. 
a)

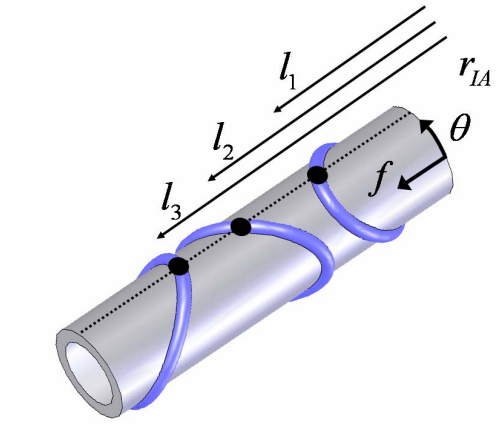

b)

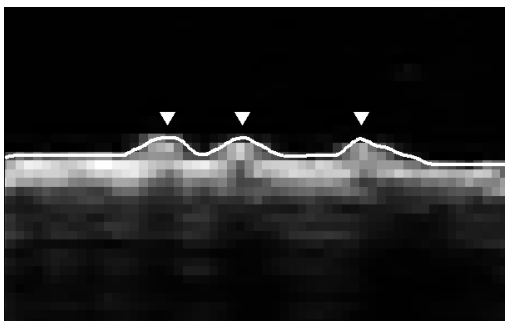

Fig. 3 - Instrument marker. a) diagram showing marker structure and measurement of $l$, b) 2D ultrasound image slice showing marker cross section, surface contour, and ridge locations.

Thus, detecting the instrument shaft delivers four out of the six degrees of freedom necessary to define $T_{M}^{I}$.

Given a series of $m$ image points along the instrument shaft arranged into an $m \times 3$ matrix $q_{I}$, the singular value decomposition (SVD) is an effective method of measuring $z_{A}$, as described in [11][12]. Translating the image points such that their mean lies at the origin, the SVD returns the principal axes of variance for the series of points,

$$
U S V^{T}=q_{I}-\frac{q_{I} u}{3}, \quad u=\left[\begin{array}{l}
1 \\
1 \\
1
\end{array}\right] .
$$

$V$ is a $3 \times 3$ orthogonal matrix, where each column describes a principal axis of variance, and $S$ is an $m \times 3$ diagonal matrix, where each element describes the magnitude of variance along the corresponding axis in $V$. The axis of the instrument shaft is taken to be the column of $V$ corresponding to the largest value of $S$.

The origin $r_{I A}$ of the intermediate frame $A$ is determined by finding the two points where the shaft axis vector, passing through the mean of $q_{I}$, intersects the image boundary and choosing the one at which the instrument enters the image. Choosing the origin of $A$ in this way provides a useful reference point for analysing the instrument marker.

The instrument shaft is detected by thresholding the 3D ultrasound image and assuming that all pixels with intensity greater than the threshold correspond to the instrument. The instrument is also assumed to always be in a configuration such that it is pointed away from the ultrasound scan head. Thus, the origin $r_{I A}$ of the intermediate frame $A$ is identified by finding the point of intersection between the shaft axis and the image boundary closest to the scan head.

\section{B. Instrument Marker}

The instrument marker consists of two parts, a cylindrical sleeve that fits over the shaft of the instrument and ridges of constant height and width fixed to the outer surface of the sleeve, as shown in Figure 3a and described in [13]. On the sleeve's surface, the ridges trace out prescribed paths, referred to as the marker shape.

The transformation $T_{M}^{A}$ is estimated from the ridge locations corresponding to the line traced along the top of the instrument marker. In an ultrasound image, the marker ridges appear as raised features along the surface of the instrument. A vertical 2D slice from the 3D image volume passing through the centerline of the instrument shaft produces a cross sectional image of the marker surface and ridges, shown in Figure $3 b$.

As shown in Figure 3a, the $z_{A}$ coordinates of the $n$ ridges are combined in a vector $l=\left[l_{1}, l_{2}, \ldots, l_{n}\right]^{T}$. This vector is related to the marker pattern through $\theta$ and $t$ by

$$
\left[\begin{array}{c}
l_{1}(\theta, t) \\
l_{2}(\theta, t) \\
\vdots \\
l_{n}(\theta, t)
\end{array}\right]=\left[\begin{array}{c}
f_{1}(\theta) \\
f_{2}(\theta) \\
\vdots \\
f_{n}(\theta)
\end{array}\right]+t u, \quad u=\left[\begin{array}{c}
1 \\
1 \\
\vdots \\
1
\end{array}\right] .
$$

The components of the vector $f(\theta)$ are smooth functions describing the $z_{M}$ coordinates of the marker ridges versus rotation angle $\theta$ about $z_{M}$. The term $t$ is the magnitude of $r_{A M}$, the vector describing the origin of marker frame $M$ with respect to the intermediate frame $A$, as shown in figure $2 b$.

Solutions for $\theta$ and $t$ are found by the following procedure. First, note that $t$ can be expressed explicitly in terms of $\theta$ by

$$
t=u^{T}(l-f(\theta)) / n .
$$

The error vector $l-f(\theta)-t u$ can then be expressed solely in terms of $\theta$ using (7) and we take its minimum norm solution,

$$
\theta=\arg \min _{0 \leq \alpha<2 \pi}\left\|l-f(\alpha)-\frac{u^{T}(l-f(\alpha))}{n} u\right\| .
$$

\section{IMPLEMENTATION}

A robotic system was constructed to validate the ultrasoundbased servoing techniques. The instrument consists of a 1.5 $\mathrm{mm}$ diameter hollow steel cannula attached to a $5 \mathrm{~mm}$ diameter hollow steel shaft. The instrument marker is attached to a $5 \mathrm{~mm}$ diameter sheath of rubber tubing, which is passed over the cannula such that the marker lies near its tip.

The instrument is submerged in a water tank and imaged 


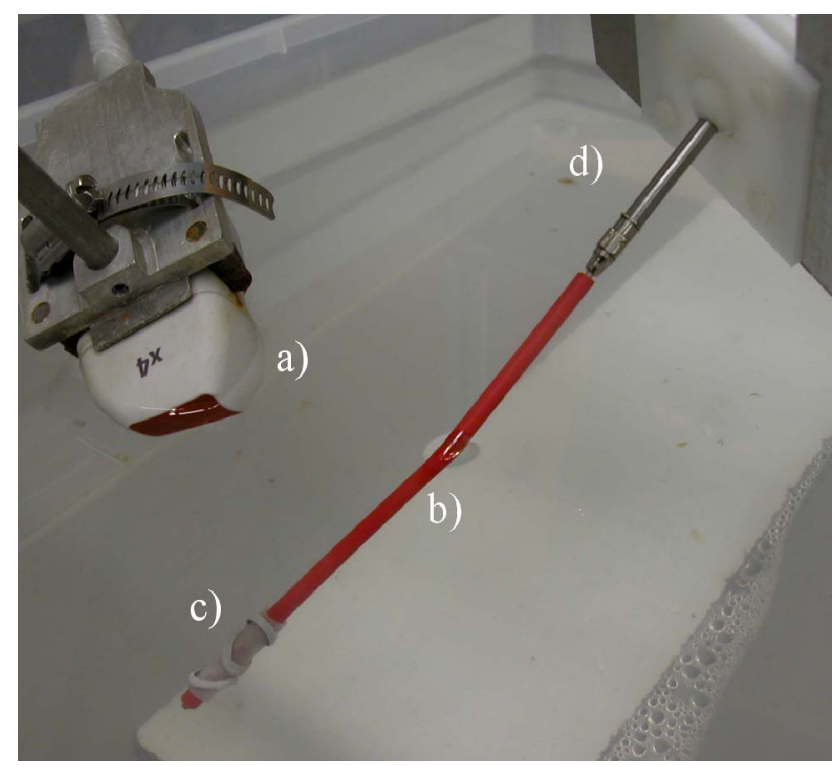

Fig. 4 - Experimental system. a) ultrasound scan head, b) instrument, c) instrument marker, d) spherical bearing. The PHANToM robot arm is not shown.

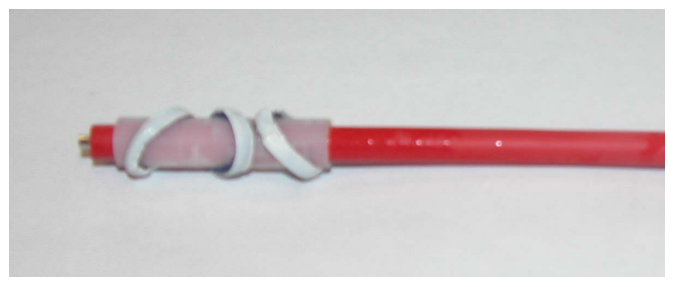

Fig. 5 - Close-up of instrument showing marker structure.

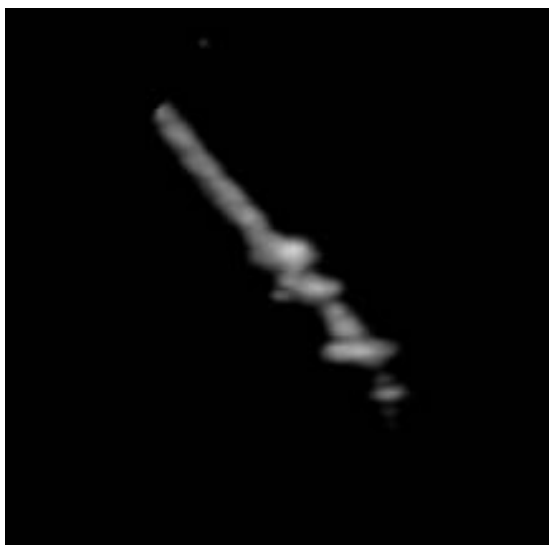

Fig. 6 - 3D ultrasound image of instrument showing marker ridges.

with a rigidly mounted 3D ultrasound scan head (SONOS 7500, Philips Medical Systems). Image resolution is $\sim 0.9$ $\mathrm{mm} /$ pixel and the image extends $150 \mathrm{~mm}$ from the scan head. A spherical bearing, representing the incision at which a real instrument enters the body, supports the instrument, which is then manipulated outside the tank by a PHANToM robotic arm (PHANToM Premium 1.5, Sensable Technologies). The experimental system is shown in Figure 4. Figure 6 shows a 3D ultrasound image of the instrument and marker.

The robot arm is controlled by a Pentium $43 \mathrm{Ghz}$ computer. 3D ultrasound images are sent to the computer and analyzed in real-time to generate error signals for the robot. Proportional and derivative control is used for position control. In addition, the robot was commanded along a constant-velocity path to prevent excess motion given large changes in position error. The path is described by

$$
p_{s}(i+1)=p_{s}(i)+v T \frac{p_{W}-p_{s}(i)}{\left\|p_{W}-p_{s}(i)\right\|},
$$

where $v$ is the instrument velocity, $T$ is the sampling interval, $p_{W}$ is the position of the robot wrist, and $p_{s}(i)$ is the intermediate waypoint for the robot wrist at update $i$.

Since image analysis requires more processing than robot control and image collection, the three subsystems run in separate asynchronous threads. The ultrasound system operates at 25 frames $/ \mathrm{sec}$; the robot operates at $1 \mathrm{kHz}$; and image analysis operates at $1.5 \mathrm{~Hz}$.

\section{A. Instrument Marker}

The marker's cylindrical body is constructed with polycarbonate plastic by a rapid prototyping process. This enables shallow grooves to be located precisely on the outer surface, in which $1 \mathrm{~mm}$ diameter hollow plastic tubing is glued to form the ridges. These tubes are then coated with echogenic material (duct tape) for improved visualization. Marker dimensions are as follows: body inner diameter $5 \mathrm{~mm}$, body outer diameter $7 \mathrm{~mm}$, and overall marker diameter $8 \mathrm{~mm}$.

The marker shape consists of three sinusoidal ridges of equal amplitude, but separated in phase by $2 \pi / 3 \mathrm{rad}$. These ridges are separated by a minimum of $3 \mathrm{~mm}$ for a total marker length of $25 \mathrm{~mm}$,

$$
f(\theta)=\left[\begin{array}{c}
3.48 \sin (\theta+4 \pi / 3)+21.52 \\
3.48 \sin (\theta+2 \pi / 3)+12.5 \\
3.48 \sin (\theta)+3.48
\end{array}\right] .
$$

As described in section IIb, markers are analyzed by first finding a vertical image plane showing a lengthwise cross section of the marker body and ridges. This image is then rotated so that the line of pixels corresponding to the surface of the instrument and marker is horizontal. A sub-image consisting of a strip centered on the instrument surface is then extracted and super-sampled by a factor of 2 using linear interpolation. The contour of the instrument surface is found by a threshold edge finding technique and filtered. Finally, the ridge locations are determined from this contour. Figure $3 \mathrm{~b}$ shows a surface contour with the corresponding ridge locations.

\section{EXPERIMENTS}

Three experiments were performed to determine the demonstration system's tracking error. The first determined 
the error as a function of image location. To this end, the instrument was driven to a series of locations within the image. The standard deviation of the instrument location in image coordinates as a function of time was recorded after the instrument had reached each location. The image locations and standard deviation values for each are shown in Table 1. Figure 7 also shows a sample instrument trajectory through the image. Note that the robot wrist will follow a nominally straight path to $p_{W}$, according to (9). As a result, the instrument will not follow a straight path since it is constrained by the spherical bearing.

Table 1 - Position control standard deviation

\begin{tabular}{|c|c|c|c|c|c|c|}
\hline \multicolumn{3}{|c|}{ Image Position (mm) } & \multicolumn{4}{|c|}{ Error Standard Deviation (mm) } \\
\hline $\mathbf{x}$ & $\mathbf{Y}$ & $\mathbf{z}$ & $\mathbf{x}$ & $\mathbf{y}$ & $\mathbf{z}$ & Mag. \\
\hline 28 & 50 & 100 & 0.37 & 0.32 & 0.28 & 0.56 \\
\hline 48 & 30 & 100 & 0.05 & 0.17 & 0.09 & 0.20 \\
\hline 48 & 50 & 100 & 0.24 & 0.45 & 0.34 & 0.61 \\
\hline 48 & 70 & 100 & 0.25 & 0.35 & 0.43 & 0.61 \\
\hline 48 & 50 & 50 & 0.44 & 0.50 & 0.46 & 0.81 \\
\hline
\end{tabular}

The second experiment determined the system's robustness to variations in probe orientation. In this case, the instrument was rotated about its axis while being held at a constant image location $(x=48 \mathrm{~mm}, y=50 \mathrm{~mm}, \mathrm{z}=85 \mathrm{~mm})$. Although the scan head was fixed throughout the experiment, rotating the instrument is equivalent to reorienting the scan head about the instrument axis. Standard deviation of instrument location in image coordinates was recorded at 4 rotation angles over approximately $180^{\circ}$. Figure 8 shows a plot of measured instrument position standard deviation versus instrument rotation angle.

The third experiment determined the system's sensitivity to instrument velocity. In this experiment, the instrument traversed three times from a starting point at the top of the image ( $x=40 \mathrm{~mm}, y=50 \mathrm{~mm}, z=50 \mathrm{~mm})$ to a target in the middle $(\mathrm{x}=40 \mathrm{~mm}, \mathrm{y}=50 \mathrm{~mm}, \mathrm{z}=90 \mathrm{~mm})$, each time at a different velocity. Figure 9 shows a plot of the instrument trajectory for each traversal. Since the instrument was manually positioned before each, there is some variation $(<10 \mathrm{~mm})$ in the actual starting points.

\section{DISCUSSION}

The experimental results validate the effectiveness of the techniques presented. They indicate that the demonstration system presented shows a viable alternative to other methods of image-based servoing. Over a range of $50 \mathrm{~mm}$, the system is able to control a surgical instrument at a target point with less than $1 \mathrm{~mm}$ of error. The error also remains constant over the range of ultrasound scan head orientations that might be observed during a surgical procedure.

The experimental results show that $\sim 3 \mathrm{~mm} / \mathrm{sec}$ is an appropriate instrument velocity, given positioning error $<0.81$
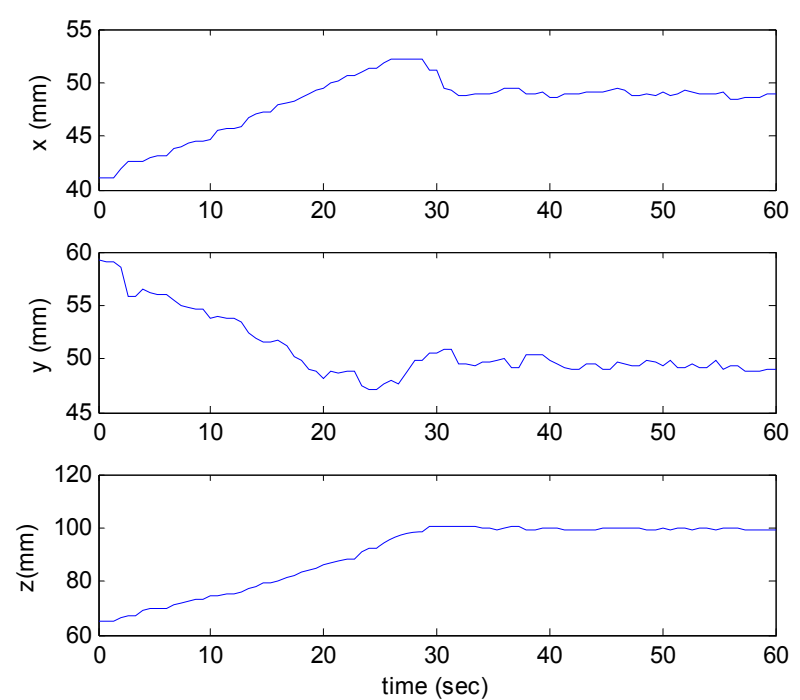

Fig. 7 - Sample instrument trajectory at $\sim 1 \mathrm{~mm} / \mathrm{sec}$.

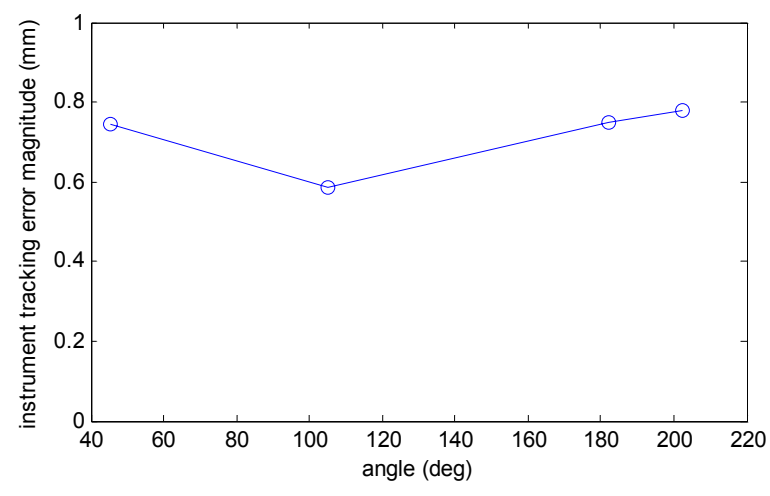

Fig. 8 - Instrument tracking error vs. instrument rotation angle
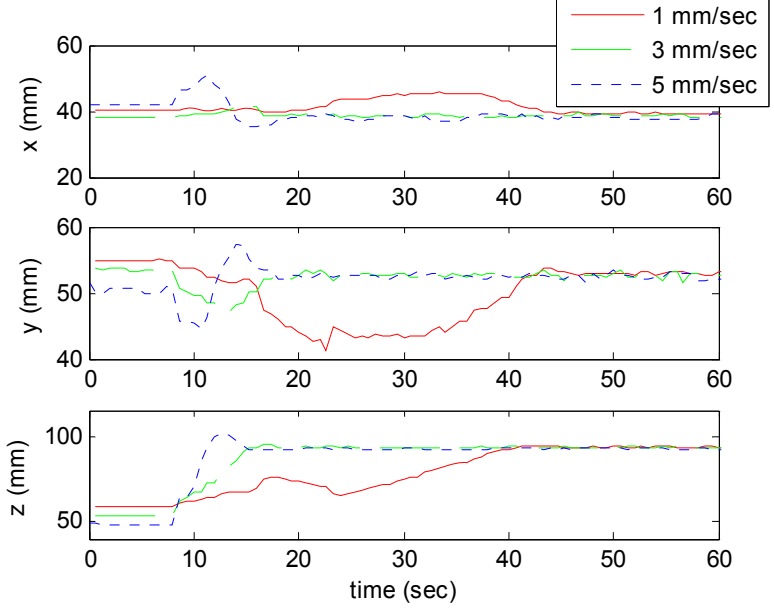

Fig. 9 - Instrument trajectory vs. instrument velocity.

$\mathrm{mm}$ and a $2 \mathrm{~Hz}$ update rate. Although steady state error was not affected by higher instrument velocity, a noticeable overshoot in $\mathrm{z}$-axis position error was observed during translation. Since overshoot can lead to inadvertent contact 
with delicate tissue structures, it is to be avoided. With faster update rates, a higher maximum velocity will likely be possible.

Future development will address the following concerns. In some cases, a small $(<5 \mathrm{~mm})$ steady-state error was observed between the actual instrument position and the target point. This is most likely due to a poor estimate of the location of the spherical bearing. An on-line estimation technique would likely produce better accuracy. It was also observed that, at the low resolution of the ultrasound system, the marker ridges became indistinguishable if they were too close together. Although this occurred over only a small angular range, not within the $40-200^{\circ}$ range shown in figure 8 , it still added significant error. When two ridges become indistinguishable, the ridge-finding algorithm can incorrectly identify image artifacts or noise as a third ridge, leading to arbitrarily large tracking error. Data filtering, improved marker designs, or higher image resolution likely will be able to correct this. Finally, large deviations in $\mathrm{x}$ and $\mathrm{y}$ are observed at low velocities. This is due to position error being calculated relative to the target point as opposed to the instrument path. Thus, deviations in $\mathrm{x}$ and $\mathrm{y}$ were not strongly corrected since the primary component of error was in the z-direction. The introduction of instrument waypoints would address this problem.

In conclusion, an autonomous real-time 3D ultrasoundguided robotic system for servoing surgical instruments has been presented and validated. This system requires no active tracking equipment and is not sensitive to arbitrary reorientation of the ultrasound probe.

\section{REFERENCES}

[1] Cannon J, R. Howe, P. Dupont, J. Triedman, G. Marx, and P. del Nido: Application of Robotics in Congenital Cardiac Surgery, Seminars in Thoracic and Cardiovascular Surgery: Pediatric Cardiac Surgery Annual. 6(1):72-83, 2003.

[2] Abolmaesumi P, S. Salcudean, W. Zhu, M. Sirouspour, and S. DiMaio: Image-Guided Control of a Robot for Medical Ultrasound, IEEE Trans. Robotics and Automation. 18(1):11-23, Feb. 2002.

[3] Hong $\mathrm{J}$ et al.: An Ultrasound-driven Needle-insertion Robot for Percutaneous Cholesystectomy, Phys. Med. Biol. 49:441-455, 2004.

[4] Vitrani M, G. Morel, and T. Ortmaier: Automatic Guidance of a Surgical Instrument with Ultrasound Based Visual Servoing, Proc. ICRA 510$515,2005$.

[5] Breyer B. and I. Cikes: Ultrasonically Marked Catheter - A Method for Positive Echographic Catheter Position Identification, Med. \& Biol. Eng. \& Comp., vol. 22, pp. 268-271, 1984.

[6] Vilkomerson D. and D. Lyons: A System for Ultrasonic BeaconGuidance of Catheters and Other Minimally-invasive Devices, IEEE Trans. Ult. Ferro. \& Freq. Cont., vol. 44, no. 2, pp. 496-504, 1997.

[7] Merdes, C. and P. Wolf: Locating a Catheter Transducer in a ThreeDimensional Ultrasound Imaging Field, IEEE Trans. Biomed. Eng. Dec. 2001

[8] Lindseth F, G. Tangen, T. Lango, and J. Bang: Probe Calibration for Freehand 3-D Ultrasound. Ultrasound in Med \& Biol. 29(11):16071623,2003

[9] Leotta D.: An Efficient Calibration Method for Freehand 3-D Ultrasound Imaging Systems. Ultrasound in Med. \& Biol. 30(7):999-1008, 2004.

[10] Stoll J., P. Dupont, and R. Howe: Ultrasound-based Servoing of Manipulators for Telesurgery, Proc. SPIE, vol. 4570, pp. 78-85, 2002.

[11] Novotny P, J Cannon, and R Howe: Tool Localization in 3D Ultrasound Images, MICCAI LNCS 2879, Springer-Verlag, Berlin, 2003.

[12] Draper K., C. Blake, L. Gowman, B. Downey, and A. Fenster: An Algorithm for Automatic Needle Localization in Ultrasound-guided Breast Biopsies, Medical Physics, vol. 27, pp. 1971-9, 2000.

[13] Stoll J. and P. Dupont: Passive Markers for Ultrasound Tracking of Surgical Instruments, MICCAI LNCS 3750, Springer-Verlag, Berlin, 2005. 